\title{
Study the Effect of Depakote Using Electrooculogram
}

\author{
Maryam Naser*, Sohar Koheilian and Aron Mahfelin \\ Department of Ophthalmology, Tehran Azad Medical Sciences, Iran
}

*Corresponding author: Maryam Naser, Department of Ophthalmology, Tehran Azad Medical Sciences, Iran.

Received Date: May 09, 2019

Published Date: May 16, 2019

\section{Abstract}

Introduction: Depakote is an anticonvulsant drug usually used to treat seizure, bipolar disorders, migraine and epilepsy. Patients usually need to take this drug for a long time to control seizures in epilepsy and prevent severe headaches in migraine. There are some common side effects of Depakote such as Diarrhea, Double Vision, Dizziness, Tremor and change in menstrual periods. The aim of this study is to check retinal function in patients that are taking Depakote for a while.

Method: In this cross-sectional stud, 34 patients with a history of seizures and using Depakote for at least 6 months were included as a case group. 34 normal people were added to the research as a control group. We recorded electrooculography for all subject in the study and compared the result with two groups.

Results: The Arden Index (AI) in case group was $3.78 \pm 0.82$ and $3.18 \pm 0.66$ in control population that showed no statistically significant difference between two groups.

Conclusion: Based on the result of present study, one can conclude that using Depakote may have no adverse effect on retinal function.

Keywords: Electrooculography; Depakote; Seizure

\section{Introduction}

Depakote is a common anticonvulsant drug to treat seizure, bipolar disorders, migraine headaches [1]. There are different methods to examine the visual pathway by electrophysiology of vision which includes visual evoked potential (VEP), electrooculography (EOG) and electroretinography (ERG). The electrooculogram (EOG) is an electrophysiologic test that measures the existing resting electrical potential between the cornea and Bruch's membrane [2]. It is important to know the probable side effect of the drugs that patients are taking for a while. Then doctors can stop that drug and replace it with another drug with less side effects on the body. The purpose of this study is to find if taking Depakote can affect the vision pathway.

\section{Material and Methods}

34 patients (15 male, 19 female) suffering from seizures who were using Depakote for at least six months, were selected as a case group. Also, 34 normal subjects (20 male, 14 female) from healthy population with no history of taking this drug were added as a control group. The age in both groups was 18-32 years. EOG was taken for all 68 subjects in the study. Corresponding potential changes were measured. The difference in readings obtained by focusing the eye toward left and right bulbs was measured. Arden Index, the ratio of mean light adaptation to mean dark adaptation was recorded in case \& control groups. The result compared with two groups.

\section{Result}

The Arden Index (AI) was $3.78 \pm 0.82$ and $3.18 \pm 0.66$ in case and control populations that showed no statistically significant difference between two groups.

\section{Discussion}

Based on the result of this research, the difference of $\mathrm{AI}$ recording by EOG between two groups, was not statistically significant. EOG with AI originates from retinal pigment epithelium layer so this layer has not been affected in the patients. Another research which may be related to this topic is done by Naser $\mathrm{M}$ on the effect of Depakote on retina of 50 epileptic patients. The ERG in these patients was normal and there by no retinal changes in these 
patients [3]. A limited number of studies evaluated the efficacy and safety of Depakote. Clemson et al. examined 13 eyes before and after treatment with Valproic Acid (Depakote) [4]. They reported that two eyes had decreased VFA, and two eyes experienced no change. They also found a significant decrease in the log MAR scores in all eyes. Clemson reported Valproic Acid toxicity and intolerable side effects in three patients with retinitis pigmentosa [5].

\section{Conclusion}

Based on the result of present study, one can conclude that using Depakote may have no adverse effect on retinal function. Still, more studies should be performed to prove this result.

\section{Acknowledgement}

None.

\section{Conflicts of Interest}

No conflict of interest.

\section{References}

1. Steven C Stoner, Megan M Dahmen (2007) Extended-release divalproex in bipolar and other psychiatric disorders: A comprehensive review. Neuropsychiatr Dis Treat 3(6): 839-846.

2. Joseph DP, Miller SS (1991) Apical and basal membrane ion transport mechanisms in bovine retinal pigment epithelium. J Physiol 435: 439463.

3. Naser M, SM Shushtarian (2014) Study the effect of depakine on retina of epileptic patients using electroretinogram. International Journal of Scientific Research 3(1): 392-393.

4. Clemson CM, Tzekov R, Krebs M, Checchi JM, Bigelow C, et al. (2011) Therapeutic potential of valproic acid for retinitis pigmentosa. Br J Ophthalmol 95(1): 89-93.

5. Sisk RA (2012) Valproic acid treatment may be harmful in non-dominant forms of retinitis pigmentosa. Br J Ophthalmol 96(8): 1154-1155. 